\title{
Title: Methods to study centrosomes and cilia in Drosophila
}

Authors: Swadhin Chandra Jana ${ }^{1, *}$, Susana Mendonça ${ }^{1}$, Sascha Werner ${ }^{1}$, Monica Bettencourt-Dias ${ }^{1, *}$

Affiliations: 1. Instituto Gulbenkian de Ciência, Rua da Quinta Grande, 6. 2780-156. Oeiras, Portugal

*- co-corresponding authors

Corresponding address:

Swadhin Chandra Jana: swadhin1cal@gmail.com

Monica Bettencourt-Dias: mdias@igc.gulbenkian.pt 


\section{Summary:}

Centrioles and cilia are highly conserved eukaryotic organelles. Drosophila melanogaster is a powerful genetic and cell biology model organism, extensively used to discover underlying mechanisms of centrosome and cilia biogenesis and function. Defects in centrosomes and cilia reduce fertility and affect different sensory functions, such as proprioception, olfaction, and hearing. The fly possesses a large diversity of ciliary structures and assembly modes, such as motile, immotile and intraflagellar transport (IFT)-independent or IFT-dependent assembly. Moreover, all the diverse ciliated cells harbor centrioles at the base of the cilia, called basal bodies, making the fly an attractive model to better understand the biology of this organelle. This chapter describes protocols to visualize centrosomes and cilia by fluorescence and electron microscopy.

Key Words: Drosophila, centrosome, basal body, cilia, intraflagellar transport, sensory neuron, sperm 


\section{Introduction:}

Centrioles are microtubule (MT)-based structures that are required to form two highly conserved eukaryotic organelles: centrosomes and cilia. The centrosome acts as major microtubuleorganizing center in cycling as well as several differentiated cells. The cilium is a MT-based protrusion that provides differentiated cells the ability to move, stir particles around and respond to various external signals. These organelles are altered in several human diseases, such as microcephaly, cancer and ciliopathies (for review [1, 2]). Therefore, a better understanding of the structure and physiology of these organelles is needed. In the last decades, the biology of these two organelles was studied in many model organisms, including Chlamydomonas, C.elegans, Drosophila, zebrafish and mouse [3-7].

The fruit fly, Drosophila melanogaster, is extensively used to investigate the fundamental mechanisms of many biological processes including the development of organ and tissues, cell division, organelle biology and animal behavior [8-11]. The fruit fly is also an excellent genetic and cell biology model organism for studying the molecular mechanisms in centrosomes and cilia biology using fluorescent microscopy and electron microscopy for a variety of reasons highlighted below [12-15].

- Most of the Drosophila proteins that are required for centrosomes and cilia biogenesis are conserved among eukaryotes, including humans (Table 1), making the studies in the fly highly relevant [16-18].

- Unlike many other models (e.g., zebrafish, mice), the fruit fly mutants of centrosomal and ciliary proteins are not embryonic lethal [19]. Thus, it is possible to investigate the role of centrosomes and cilia in organ and tissue development in a whole animal by eliminating these organelles. 
- Drosophila possesses cells that harbor both immotile cilia (9+0: e.g., in external sensory neurons) and motile cilia (9+2 in sperm). Ciliary functions can be tested in the fly by measuring the response to sensory stimuli. This can be done directly in physiological assays at the level of sensory neurons (e.g. calcium imaging, electrophysiology) or in a more indirect manner by quantifying behavioral responses. An obvious read-out for cilia function in sperm is fertility. In embryo, larvae and adult, the sensory reception is mediated by cilia grown on type-I sensory neurons of the peripheral nervous system. Type-I sensory neurons can grossly be divided into two categories: 1) external sensory neurons (that grow 9+0 type axonemes without dynein arms) and 2) chordotonal neurons (that grow 9+0 type axonemes with dynein arms). All different external sensory neurons grow structurally and functionally diverse cilia (Figure 1-3, 5) [15, 20]. Notably, all cilia on sensory neurons require intraflagellar transport (IFT) for their assembly [21]. Furthermore, Drosophila testes harbor sperm cells and their precursors that also grow cilia. While the cilia grown in sperm cells are motile $(9+2)$, sperm precursor cells (also called spermatocytes) have immotile cilia $(9+0 / 1$, Figure $1,4,6)[22,23]$. The cilia in testes assemble in an IFT-independent manner [21]. Many structurally and functionally diverse cilia can be found in different uniand multi-cellular animals. This diversity can be represented by one single model organism, Drosophila [24]. Additionally, cilia assembly and maintenance in many organisms are regulated by complex combinations of IFT- and non-IFT-dependent transports which are hard to distinguish [25]. Therefore, the fly is particularly suitable to distinguish IFT-related functions from other cilia-associated functions of any centrosomal and ciliary molecule.

- During the development and differentiation of all ciliated cells, the centrosome assembles, centriolesmigrates to the apical parts of the cell where it gets anchored and modified, and grows a cilium [2]. Additionally, in Drosophila mature sperm cells the centrosome loses most of its components (centriolar components as well as pericentriolar material (PCM)). 
This process is called centrosome reduction [26]. Thus, studies in the neurons and testes can allow one to address multiple aspects of "the life of a centrosome" such as centriole duplication, centriole stability, centriole elongation, centriole separation, PCM recruitment and removal, and ciliogenesis among others.

- Drosophila harbors different types of centrosomes. They vary in their length and the types of microtubules they are made of. For example, centrioles in the embryo are short and made of nine doublet microtubules, whereas those in sperm cells are uniquely long and consist of nine triplet microtubules (Figure 4, 6) [27]. Long centrioles in sperm allow rapid and easy analysis of this organelle by imaging, providing an appropriate system to understand the mechanisms underlying centriole elongation $[22,28]$. Studies in centriolar cells with or without cilia in the embryo are widely appreciated [28]. Moreover, dissection and imaging of the tissues that have ciliated cells in larvae, pupae, and adults are simple (Figure 2) [20, 29].

- Drosophila is a widely preferred model organism for biological studies due to its short generation time and ease to do genetics. Importantly, mutants and RNAi lines for most of the known centriolar and ciliary components are available (Table 1). Flies that express fluorescently tagged centrosomal and ciliary proteins are available to the community and several of those fluorescently tagged components are expressed under the control of endogenous promoters. In addition, antibodies against many of those proteins are available (see Table 1).

In this chapter, we describe protocols to visualize centrosomes and cilia directly or by immunofluorescence either in Type-I sensory neurons or in testes in both pupae and adults. Moreover, we provide detailed protocols to perform electron microscopic analyses of centrosome and cilia ultrastructure in different ciliated cells in pupae or adult. Finally, we show and discuss results and limitations of using those protocols. 


\section{Visualization of centrosomes and cilia using fluorescence microscope}

Centrosomes and cilia can be studied at all different stages of Drosophila development: in embryos, larvae, pupae, or adults [28-30]. In pupae and adults, the cuticles that encapsulate the sensory neurons are opaque and display autofluorescence making antibody staining and fluorescence live imaging difficult. Recently, several methods for studying centrosome and cilia in sensory neurons in pupae/adults were reported [15, 20]. Here, we present protocols (see section 3.1) to visualize cilia by live-imaging of pupal/adult tissues, such as different antennal segments, maxillary palp, proboscis, wing and leg (Figure 2). We also present protocols (see section 3.1 and 3.2) for immunostaining of sensory neurons (Figure 3) and sperm cells (Figure 4) in pupal/adult antenna and testes, respectively. Protocols to study these organelles in embryo and larvae, and for live imaging of the centrosome in testes can be found elsewhere [29, 31, 32].

The Drosophila second antennal segment harbors approximately 500 sensory neurons called chordotonal neurons, which grow cilia and are required for hearing and coordination in the adult flies [33]. Several markers can be used to study the different centrosome or ciliary compartments in sensory neurons, (Table 1, Figure 3A). For example, the pericentriolar material and ciliary axoneme can be specifically labeled using antibodies that recognize pericentrin-like protein (PLP) and acetylated tubulin, respectively (Figure 3C). Here, we describe two methods of fixation of antenna for immunostaining of the chordotonal neurons. Although we do not find remarkable differences using both protocols in the localization of the studied components, we did not systematically compare large set of centrosome and cilium components. Many antibodies and flies expressing several fluorescent reporters can be used to label specific compartments of the centrosomes (e.g, centriole and pericentriolar material) and cilia (e.g., transition zone, proximal segment of the cilia, ciliary dilation, distal segment of the cilia, IFT-components etc.) (Figure 3A; for detailed list see Table 1). Since, all the ciliated sensory neurons present in adult Drosophila develop during the pupal stage, and these neurons are assumed to be long-lived, the techniques 
discussed here (section 3.1) can be used to study the assembly and maintenance of these organelles $[15,20]$. However, a live-imaging protocol to track the same set of ciliated sensory neurons in pupal stage or adult for several days is still unavailable. Such tool would help to better understand the centrosomes and cilia biology.

In testes, a stem cell divides to form a cyst cell and a gonial cell. The gonial cell subsequently undergoes four rounds of incomplete mitotic divisions to produce a cyst of 16 primary spermatocytes (Figure 4A). Primary spermatocytes go through a long G2 phase, during which two pairs of small centrioles $(0.2 \mu \mathrm{m})$ elongate strongly until they reach about eight times their initial size $(1.5 \mu \mathrm{m})$ and grow small protrusions that are structurally similar to primary cilia (Figure 4A; section 3.2). In spermatocytes and sperm cells, these organelles can be labeled using PACT::GFP (marks centrioles) and acetylated tubulin (marks centrioles and cilia) (Figure 4C; for a detailed list of markers used in sperm cells see Table 1). The protocols mentioned here can be used to analyze centrioles and cilia in the mutants that affect the assembly, conversion and stability of these organelles [22]. However, to better understand the morphogenesis and functional transformations of these organelles in sperm cells it would be desirable to develop protocols for live imaging of centrosomes and cilia during spermatogenesis.

\section{Ultrastructure of centrosomes and cilia in Drosophila}

Centrioles are small MT-based cylinders with $~ 250 \mathrm{~nm}$ in diameter and 300-500 nm in length, while cilia are MT-based long cylinders with 250 nm in diameter and variable lengths [1, 2]. Thus, many of the centriole and cilium substructures are under the resolution limit of the light microscope, so a detailed ultrastructural analysis is critical to understand their mechanisms of assembly and maintenance. Although, the protocol to study centrosomes and cilia ultrastructure using transmission electron microscopy in embryo and larvae is not yet well established, these 
organelles have been investigated by several groups in the pupal/adult antenna and testes [15, $20,22]$ (also see section 3.3).

Due to the special 3D-orientation of the neurons in the second antenna segment (see Figure 3A), both cross- and longitudinal sections should be analyzed to study centrosomes and cilia. Notably, longitudinal and cross sections of antennae can be used to study the cross and longitudinal section of the centrosomes/cilia, respectively (Figure 5 A) [29]. Each chordotonal neuron grows a sensory cilium (Figure 5B). Each cilium has different components, such as a long rootlet, two basal bodies, a transition zone, a proximal segment of the cilia, a ciliary dilation, and a distal segment of the cilia (Figure 5C). The proximal part of the chordotonal cilium consists of radially arranged nine doublet microtubules with dynein arms which are required for the function of the cilium, but is devoid of central pair of singlet microtubules $(9+0)$ (Figure $5 \mathrm{C})$. However, little is known about the ultrastructure of the basal bodies and cilia in different sensory neurons.

Centrosomes and cilia undergo multiple morphological and functional transformations during spermatogenesis (Figure 4A). In spermatocytes, centrioles/basal bodies are formed of nine triplet microtubules, whereas primary cilia exhibit either nine doublet microtubules $(9+0)$ or nine doublet microtubules with a central singlet microtubule (9+1) (Figure 6; for protocol see section 3.3). These small primary-like cilia are devoid of dynein arms and hence considered immotile. Each spermatocyte then undergoes two consecutive meiotic divisions without DNA replication and centriole duplication. As a result, in each early spermatid a single basal body (or centriole) nucleates the sperm flagellum. Interestingly, the elongating sperm flagellum that is motile consists of nine radially arranged doublet microtubules and a central pair of singlet microtubules $(9+2)[22$, 23]. However, how immotile cilia and motile flagella are assembled and how different types of cilia grow from same basal body is still unclear. 
Using the protocols (see section 3) mentioned in this chapter, it is possible to execute both live imaging of centrosomes and cilia for few minutes, and to immunodetect and perform ultrastructure analysis of these organelles. However, these organelles are small in size (especially in diameter), their assembly is slow and they are long-lived. Therefore, in the future it will be critical to develop protocols to both image these organelles using super-resolution microscopy and to perform live imaging in the ciliated cells for several days. Moreover, novel ultrastructural analysis of these organelles in different tissues are desired, where samples are prepared using high-pressure freezing techniques or studied by cryo-electron microscope.

\section{Materials:}

We prepare all solutions using deionized water (d-water, prepared using Millipore Elix 10, USA) or Milli-Q water (prepared by Millipore Synthesis 10, UAS). All reagents should be prepared and stored at room temperature (RT) (unless indicated otherwise). All waste disposal regulations must be followed when disposing of biohazardous waste materials.

\subsection{Drosophila stocks and husbandry:}

The flies of known genotype can be obtained from the Bloomington stock center, USA, Vienna Drosophila Rnai Center, Austria and National Institute of Genetics-FLY, Japan. Grow Drosophila in vials or bottles containing standard culture media that are used in the above mentioned centers. Finally, collect the specific stages of Drosophila required for the studies.

Culture medium for Drosophila: To make one liter of medium, weigh $80 \mathrm{~g}$ Molasses, $22 \mathrm{~g}$ Bett Syrup, $80 \mathrm{~g}$ Corn meal, $18 \mathrm{~g}$ Yeast granules, $10 \mathrm{~g}$ Soy flour and $8 \mathrm{~g}$ Agar-Agar in a plastic beaker. Mix all ingredients in a beaker before adding the boiling d-water. Gradually 
add $1050 \mathrm{~mL}$ boiling d-water. Transfer the mixed medium to a 2 liters Schott flask. Autoclave the medium at $121^{\circ} \mathrm{C}$ for 30 minutes. Finally, add $12 \mathrm{~mL} 15 \%$ niapagin (made in ethanol) and $8 \mathrm{ml}$ propionic acid, when the medium temperature reaches $45^{\circ} \mathrm{C}-50^{\circ} \mathrm{C}$. This recipe is used in Vienna Drosophila Rnai Center, Austria.

\subsection{Components for direct visualization and immunostaining of sensory neurons:}

1. Use halo carbon oil (Sigma, USA) to mount the tissues for live imaging.

2. Phosphate buffered saline (PBS): Dissolve phosphate buffer tablets (Sigma, USA) in dwater. According to company datasheet, when one tablet is dissolved in $200 \mathrm{~mL}$ of $\mathrm{d}$-water yields $10 \mathrm{mM}$ phosphate buffer, $2.7 \mathrm{mM}$ potassium chloride and $137 \mathrm{mM}$ sodium chloride, $\mathrm{pH} 7.4$, at $25^{\circ} \mathrm{C}$.

3. PBST-X buffer: 0.1 or $0.3 \%$ PBST-X is made by dissolving required amount of Triton $X-$ 100 in PBS solution. 0.1\% PBST-X generally should be used in antenna and testes immunostaining protocols (see section 3.1, 3.2 and Note 1).

4. Fixative solutions (for immunostaining): Mix one volume of $16 \%$ formaldehyde (methanol-free, ultrapure EM grade, Polyscience Inc, PA, USA) three volumes of PBS to make $4 \%$ formaldehyde fixative.

5. Stefanini's fixative: For $1 \mathrm{ml}$ of fixative, combine $250 \mu \mathrm{l}$ of $16 \%$ PFA with $150 \mu \mathrm{l}$ of saturated picric acid, $150 \mu \mathrm{l} 0.5$ M PIPES buffer $(\mathrm{pH} 7.6)$ and $450 \mu \mathrm{l} d$-water (supplemented with $0.1 \%$ Triton-X - see Note 2 ).

6. Use optimal cutting temperature (OCT) compound to embed the heads.

\subsection{Components for immunostaining of testes:}

1. PBS: See section 2.2 point 2. 
2. $1 \mathrm{X}$ Testes buffer: This buffer contains $183 \mathrm{mM} \mathrm{KCl}, 47 \mathrm{mM} \mathrm{NaCl}$ and $10 \mathrm{mM}$ Tris- $\mathrm{HCl}$, $\mathrm{pH} 6.8$.

3. Fixative solutions: See section 2.2 point 4.

4. Siliconized coverslips: Wash new coverslips in ethanol for 10 minutes in a shaker, Airdry and incubate in Sigmacoat solution (Sigma, USA) for 1 minute. Air-dry coverslips.

\subsection{Components for TEM sample preparation:}

1. Sodium bi-phosphate buffer: Make stock solution A and B first:

Stock solution A: $0.2 \mathrm{M}$ monobasic sodium phosphate, monohydrate (dissolve $27.6 \mathrm{~g}$ $\mathrm{NaH}_{2} \mathrm{PO}_{4}$ in $1 \mathrm{~L}$ Milli-Q water)

Stock solution B: $0.2 \mathrm{M}$ dibasic sodium phosphate (dissolve $28.4 \mathrm{~g} \mathrm{Na} 2 \mathrm{HPO} 4$ in $1 \mathrm{~L}$ MilliQ water)

Mix $28 \mathrm{~mL}$ of stock solution A with $72 \mathrm{~mL}$ of stock solution $\mathrm{B}$ to obtain $100 \mathrm{~mL}$ of $0.2 \mathrm{M}$ of phosphate buffer $\mathrm{pH} 7.2$.

2. PBS: See section 2.2 point 2.

3. Fixative solutions: $2 \%$ formaldehyde, $2.5 \%$ glutaraldehyde in $0.1 \mathrm{M}$ bi-phosphate buffer is generally used for fixing the samples. To make $100 \mathrm{~mL}$ of fixative solution, mix $12.5 \mathrm{~mL}$ of $16 \%$ formaldehyde (EM grade), $10 \mathrm{~mL}$ of $25 \%$ glutaraldehyde (EM grade), $27.5 \mathrm{~mL}$ of $\mathrm{d}$-water and $50 \mathrm{~mL}$ of $0.2 \mathrm{M}$ sodium bi-phosphate buffer.

5. Uranyl acetate (UA) solution: Use $2 \%$ UA in d-water $(20 \mathrm{~g} / \mathrm{L})$ and $2 \%$ UA in $70 \%$ methanol $(20 \mathrm{~g} / \mathrm{L})$ for sample fixation/processing and for the staining of ultra-thin sections, respectively. 
6. Use Propylene oxide (SPI supplies) for TEM sample preparation.

7. EPON ${ }^{\mathrm{TM}}$ Epoxy resin dilutions: Make stock solutions first:

Stock EPON I: Mix $62 \mathrm{~mL}$ of EMbed 812 and $100 \mathrm{~mL}$ of dodecenyl succinic anhydride (DDSA).

Stock EPON II: Mix $100 \mathrm{~mL}$ of EMbed 812 and $90 \mathrm{~mL}$ of nadic methyl anhydride (NMA)

Make the EPON working solutions according to Luft (1961): Mix $30 \mathrm{~mL}$ of EPON I with $70 \mathrm{~mL}$ of EPON II and add $1.5 \mathrm{~mL}$ of Tris-(dimethylaminomethyl) phenol (DMP-30) (accelerator) in the mixture (see Note 3).

8. Reynolds Lead citrate solution: Make $30 \mathrm{~mL}$ de-carbonated water by boiling the $\mathrm{d}$-water in a microwave and cooling it in a freshly washed sealed container. Add $30 \mathrm{~mL}$ of decarbonated water to $1.33 \mathrm{~g}$ of lead nitrate and $1.76 \mathrm{~g}$ of sodium citrate in a volumetric flask and shake vigorously for 1 minute followed by sonication for 30 minutes with shaking the container every five minutes. Add $8.0 \mathrm{~mL}$ of $1 \mathrm{~N}$ sodium hydroxide $(\mathrm{NaOH})$ to the opaque mixture. The mixture should then turn clear. Make up the volume to $50 \mathrm{~mL}$ with the decarbonated water. Store the solution in a tightly sealed volumetric flask (see Note 4).

\subsection{Instruments:}

1. Use any surgical blade (Swann Morton Ltd, UK) to cut heads from the flies.

2. Use plastic molds (Simport, Canada) or similar to make blocks with the samples for cryosectioning.

3. Use a Leica CM 3050 S Cryostate (Leica BioSystems, Germany) or similar to cut the cryo-frozen tissues of Drosophila head. 
4. Use a Dako Pen (Dako Agilent Technologies, USA) or similar to make hydrophobic boundaries on the glass slides for immunostaining.

5. Use a Leica TCS Sp5 II confocal microscope, Leica BioSystems, Germany or similar to image all the fluorescent samples.

6. Use a GenLab oven $\left(60^{\circ} \mathrm{C}\right.$ incubator) or similar to polymerize the resins.

7. Use a Hitachi Transmission Electron Microscope with AMT 2kX2k digital camera or similar to collect EM images.

\section{Methods:}

All our procedures can be carried out at room temperature (RT) unless otherwise specified.

\subsection{Direct visualization or immunostaining of centrosomes and cilia in sensory neurons in the pupae and adult:}

1. For live imaging: To study the centriole and ciliary components in live tissues, dissect required tissues, such as antenna, proboscis, wing and leg (Figure 2). Mount the samples in halocarbon oil (Sigma, USA) (see Note 5).

2. Sample preparation before sectioning:

Protocol 1: Cut the heads from adult flies using a surgical blade (Swann Morton Ltd, UK) or dissect out heads from 30 to 100 hour old pupae. Align 16-20 heads in a plastic mold containing OCT (Figure 3B). Arrange heads in an appropriate orientation (either antenna facing up or one eye facing up) to obtain the desired sections of antenna. Then freeze the mold in dry ice (see Note 6). This protocol is adapted from [20]. 
Alternatively, fix whole flies (without the wings) in $4 \%$ formaldehyde in PBST-X buffer for 1 hour at $4^{\circ} \mathrm{C}$ and mount heads in a mold as described above (see Note 7 ).

Protocol 2: Fix $\sim 16-20$ fly heads in 2 ml Stefanini's fixative for 40 minutes on ice (see Note 8). Subsequent steps should be done at RT. Wash the heads for $3 X 20$ minutes in $0.1 \%$ PBST- $X$ to remove the picric acid that might interfere with immunostaining. Incubate the fixed heads in $10 \%$ sucrose in PBST-X on a rotator for 1 hour. Then incubate heads in $25 \%$ sucrose in PBST-X and place them on a rotator for overnight at $4^{\circ} \mathrm{C}$. After removing the residual sucrose solution, embed and orient the heads in OCT as described before (see Note 7 and 9). This protocol is adapted from [34].

3. Collect sections (around 10-12 $\mu \mathrm{m}$ thick) on poly-L-lysine coated glass slides (SigmaAldrich, USA). Multiple sections can be collected on a slide and samples can be collected in two/three rows (Figure 3B). Also, optionally collect consecutive sections in two separate slides to immunostain the same sample with separate sets of antibodies.

4. Air dry the slide(s) with sections for 1 minute and make a border around the sections using a Dako pen-(see Figure 3B). This pen mark creates a hydrophobic boundary that prevents aqueous solutions from running off the slide.

5. Keep the slide(s) in a humid chamber pre-wetted with PBS for further use. Then incubate sections in the fixative solution (for IF) for $1 \mathrm{hr}$ at $4^{\circ} \mathrm{C}$ (see Note 10 ).

6. Wash sections with PBTX buffer for $4 \mathrm{X} 10$ minutes at RT.

7. Then block sections using $0.1 \%$ PBST-X $+5 \%$ bovine serum albumin (BSA) for $1 \mathrm{hr}$ at RT (see Note 1).

8. Incubate sections with primary antibody solutions (with required dilutions) made in $0.1 \%$ PBST-X $+5 \%$ BSA for overnight at $4^{\circ} \mathrm{C}$ (see Note 1$)$. 
9. Wash slides with $0.1 \%$ PBST-X $+5 \%$ BSA for $3 X 10$ minutes at RT (see Note 1 ).

10. Stain the sections with required secondary antibody solutions (with required dilutions) made using $0.1 \%$ PBST-X $+5 \%$ BSA for 2 hrs at RT (Note 1 and 11 ).

11. Wash samples with $0.1 \%$ PBST-X at RT for $3 X 10$ minutes.

12. For DNA staining, incubate sections in a DAPI ( $1 \mu \mathrm{L}$ DAPI/mL PBS) or Hoechst solution for 15 minutes at RT.

13. Wash again for 10 minutes with PBS buffer at RT.

14. Mount sections using either $70 \%$ glycerol (Sigma-Aldrich, USA) or vectashield (Vector Laboratories, USA), cover and seal the cover slips with nail polish. Alternatively, instead of staining the sample(s) using DAPI solutions (mentioned in step 12), mount samples using vectashield with DAPI (Vector Laboratories, USA). Samples are ready to be imaged using any fluorescence microscope.

\subsection{Visualizing directly or immunostaining of centrosomes and cilia in testes:}

For Dissection:

1. Dissect testes from larvae, pupae and adult males in a drop $1 \mathrm{X}$ testis buffer (see section 2.2 point 4) containing EDTA ( $2 \mu \mathrm{L} 0.5 \mathrm{mM}$ EDTA in $1 \mathrm{~mL}$ testes buffer) for immunostaining. For pupae or adult, hold the fly with the legs facing up and pull out the male genitalia (dark brown spot present below the abdomen). The testes will come out with it. If they do not, insert the forceps inside abdomen of the fly and pull out the testes. For immunostaining intact testes, ignore steps $2-5$ and fix the samples in $4 \%$ formaldehyde as described in step 6. The detailed protocols for imaging live sperm cells [32] and dissection of testes of different stages [28, 30, 32] can be found elsewhere . 
For immunostaining:

2. Transfer testes to a $4 \mu \mathrm{L}$ drop of $1 \mathrm{X}$ testis buffer placed on a poly-L-Lysine slide. Use up to 3 pairs of testes per slide and 2 slides per antibody.

3. Randomly squash testes with forceps and cover them with a $18 \times 18 \mathrm{~mm}$ siliconized coverslip (see Note 12).

4. Put slides in liquid nitrogen (see Note 13).

5. Remove coverslips by flipping them off with a scalpel.

6. For fixation: Acetone-methanol fixation: Place slides in dry ice-cold methanol in an upright slide box for 8 minutes. Then, transfer slides to dry ice-cold acetone in an upright slide box and incubate for 10 minutes.

Formaldehyde fixation: Place slides in 4\% Formaldehyde in PBS (see section 2.3 point 3) for 15 minutes at RT.

8. Make a border around the sections using Dako pen (see section 3.1 step 4).

9. Wash samples for $3 X 5$ minutes in PBS at RT.

10. Block samples in PBS $+1 \%$ BSA for $1 \mathrm{hr}$ at RT.

11. Keep slides in a humid chamber pre-wetted with PBS and incubate slides with primary antibody in PBS $+1 \%$ BSA overnight at $4^{\circ} \mathrm{C}$ (see Note 1 and 14 ).

12. Wash slides for $4 \mathrm{X} 10$ min each with PBS $+1 \%$ BSA.

13. Incubate samples with secondary antibody (with required dilution) in PBS $+1 \% B S A$ for $2 \mathrm{hr}$ at RT (see Note 11 and 14). 
14. Wash slides for 2 X 15 minutes with PBS $+1 \%$ BSA and then 1 X 15 min with PBS.

15. For DNA staining, incubate samples in a DAPI ( $1 \mu \mathrm{L}$ DAPI /mL PBS) or Hoechst solution for 15 minutes at RT.

16. Wash samples for $1 X 5$ minutes with PBS. Rinse the slides in water and mount slides in vectashield. Cover samples with a clean coverslip (non-siliconized) and seal them. Samples are ready to be imaged in any fluorescence microscope.

\subsection{Ultra-structure analysis of centrosomes and cilia in antenna and testes:}

1. Dissection: Remove heads and dissect testes from a pupae or adult in PBS as mentioned in section 3.1 step 2 and 3.2 step 1 , respectively.

2. For antenna: First, immerse the heads in fixative ( $2 \%$ formaldehyde, $2.5 \%$ glutaraldehyde in $0.1 \mathrm{M}$ sodium bi-phosphate buffer $\mathrm{pH} 7.2-7.4)$ for $30 \mathrm{~min}$. Pierce the third antennal segments using a thin tungsten needle (see Note 15). Finally, fix heads in $2 \%$ formaldehyde, $2.5 \%$ glutaraldehyde in $0.1 \mathrm{M}$ sodium bi-phosphate buffer $(\mathrm{pH} 7.2-7.4$ ) overnight at $4^{\circ} \mathrm{C}$ with rotation.

For testes: Fix whole testes in 2.5\% glutaraldehyde in PBS at $\mathrm{pH} 7.2-7.4$ for 2 hrs at $4^{\circ} \mathrm{C}$ with rotation.

3. Wash samples for $5 x 5$ minutes in sodium bi-phosphate buffer ( $\mathrm{pH} 7.2-7.4)$.

4. Post-fix heads and testes in $1 \%$ Osmium tetroxide $\left(\mathrm{OsO}_{4}\right)$, for $1 \mathrm{hr} 30$ minutes and $1 \mathrm{hr}$ on ice, respectively.

5. Wash samples with d-water for $5 \times 5$ minutes.

6. Incubate samples in $2 \%$ uranyl acetate for 20 min in RT with rotation. 
7. Wash samples in d-water for $3 \times 10$ minutes.

8. Dehydrate samples in a graded series of alcohol (50\%, 70\%, $90 \%$ and $100 \%)$ for 10 min in each solution. Repeat the dehydration in $100 \%$ alcohol for two more times to obtain complete dehydration.

9. Treat samples in propylene oxide for $2 \times 15$ minutes.

10. Incubate heads in 1:1 propylene oxide: EPON resins for $3 \mathrm{hrs}$ with rotation. For testes, incubate samples for $1 \mathrm{hr}$.

11. Incubate heads in EPON resin for overnight at $4^{\circ} \mathrm{C}$ and testes for $1 \mathrm{hr}$ at $\mathrm{RT}$.

12. Next day, incubate heads in fresh resin (same batch as used in step 11) for 2 hrs at RT with rotation. This step is not required for testes samples.

12. Align samples in the molds with resin and polymerize them overnight in $70^{\circ} \mathrm{C}$ oven.

13. Sectioning: Cut semi-thin sections $(500 \mathrm{~nm})$ using a diamond knife on an ultramicrotome and collect sections on a glass slide. Stain sections with TBO to check the gross morphology of the tissues under a compound microscope. After reaching the area of interest, cut serial ultrathin sections $(\sim 70 \mathrm{~nm})$ and collect them on a formvar-coated copper grid.

14. Uranyl acetate staining: Stain grids (with sections) by putting them (upside down) on a drop of $2 \%$ uranyl acetate in $70 \%$ methanol for 3 minutes at RT (see Note 16 ).

15. Rinse sections by passing the grid through two drops of $70 \%$ methanol.

16. Wash grids in four drops of d-water and air-dry the grids. 
17. Lead citrate staining: In a closed chamber with $\mathrm{NaOH}$ pastilles (used to absorb humidity), put the grids (upside down) on a drop of lead citrate for 1 minute (see Note 17).

18. Wash grids in five drops of d-water and air-dry the grids. The sections are ready to be imaged in a Transmission Electron Microscope (TEM).

\section{Notes}

1. For immunostaining of some epitopes (proteins) in the antenna, a higher amount of the detergent $(0.3 \%$ Triton $\mathrm{X}-100)$ can be required. Although, for immunostaining of most of the proteins in the testes, the detergent is not required, for some epitopes detergent $(0.1 \%$ Triton X-100) might be required.

2. The Triton-X in the buffer makes the fixative access the cells faster through the cuticle.

3. These two stock solutions should be stored at $4^{\circ} \mathrm{C}$ separately. Since both EPON I and EPON II are strongly hydroscopic, mix the stock solutions just before the mixture is used.

4. Since lead citrate reacts with carbon dioxide $\left(\mathrm{CO}_{2}\right)$ to form lead carbonate precipitate, the lead citrate solution should be stored in a sealed container.

5. To avoid scattering effects, carefully remove all the bubbles that may adhere to the tissue of interest.

6. Carefully remove all the bubbles adhered to the tissue of interest before freezing the sample.

7. It might be preferable to cut both the eyes as they possess pigmented cells that show strong autofluorescence and they do not harbor any ciliated cells. 
8. Heads should sink during the fixation. Occasionally shake the samples with care to help fixing the heads.

9. The incubation in sucrose helps preserving the tissue architecture. Removing the residual sucrose solution from heads helps to embed samples more evenly in the OCT, which should improve sectioning.

10. Avoid any type of shaking during the entire process of immunostaining.

11. As fluorophores tagged to secondary antibodies are light-sensitive, keep the chamber in the dark from now on.

12. Pre-siliconized coverslips should be washed in ethanol and air-dry before using (see section 2.3 point 4$)$.

13. To avoid tissue damage before fixation, do not keep slides in liquid nitrogen for more than $1 \mathrm{hr}$.

14. A volume of $50-75 \mu \mathrm{L}$ of antibody solution per slide should be enough.

15. Since olfactory neurons are encapsulated by hard cuticles in the third antennal segment, appropriate piercing of the third segment is very critical. This helps the fixative to properly infiltrate the tissue.

16. Protect the grids from the light to avoid precipitate formation.

17. Use a closed chamber with $\mathrm{NaOH}$ to protect the sections from $\mathrm{CO}_{2}$, avoiding precipitate formation. 
Figures and legends:

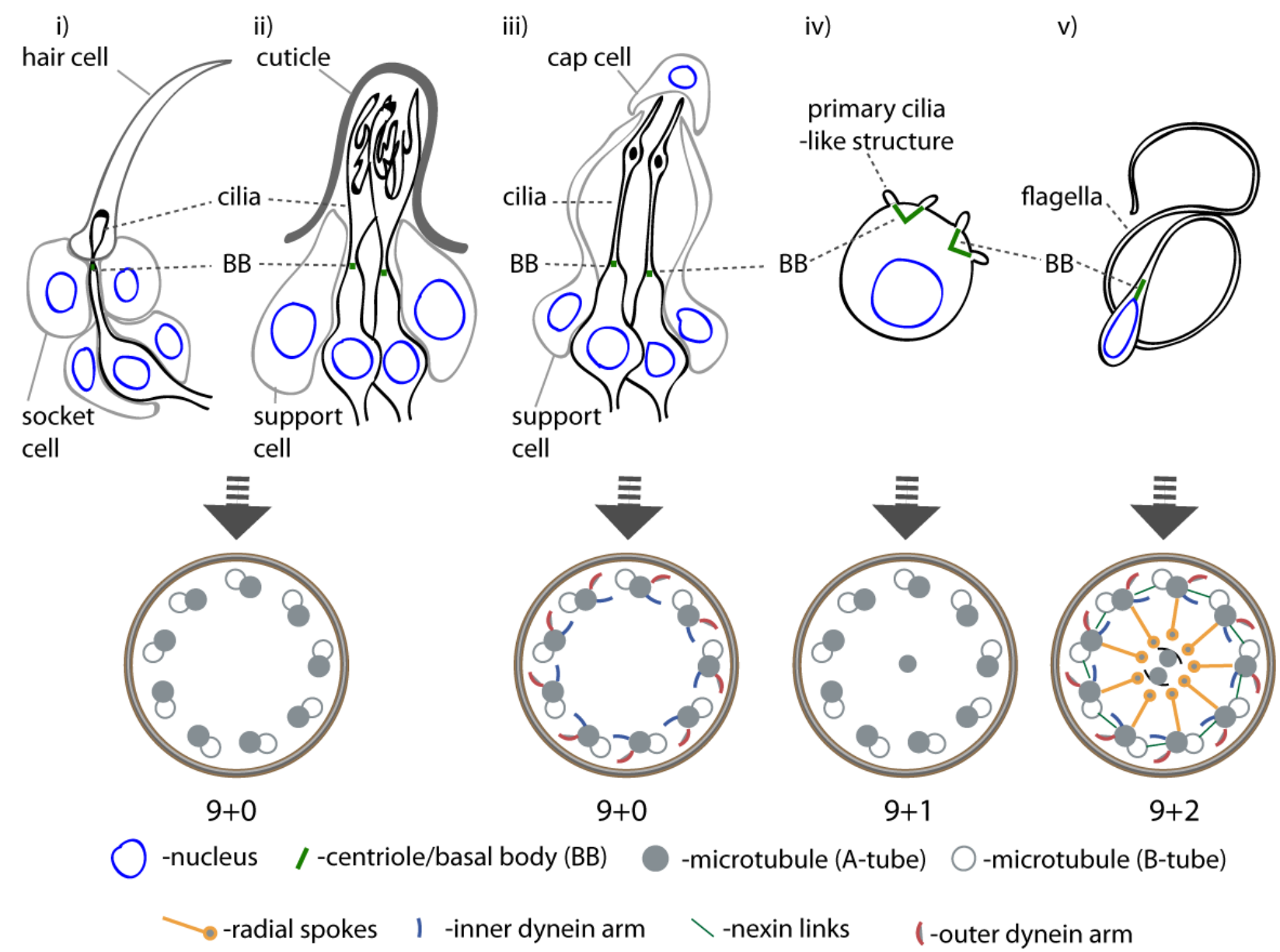

Figure1: Drosophila harbors many cells that grow different types of cilia. Schematic representation of some ciliated cells present in adult Drosophila melanogaster. Cilia grow from the distal tip of the dendrites of the Type-1 sensory neurons in the fruit fly. These are generally divided in two groups: neurons in external sensory (ES) organs (i-ii) and chordotonal organs (iii). Cilia in ES organs usually display a 9+0 microtubule organization (without dynein arms). The proximal segment of the chordotonal cilia shows 9+0 microtubule architecture with dynein arms. Thus, these cilia are believed to be motile and mutants without dynein arms suffer hearing loss. In neurons of the peripheral nervous system (PNS), cilia assembly relies on intraflagellar transport (IFT). Primary cilia-like structures and flagella are found in spermatocytes (iv) and in elongating 
spermatids (v). While primary cilia-like structures in spermatocytes (iv) display 9+0 or 9+1 MT organization, a 9+2 architecture with a central pair, radial spokes, nexin links, and dynein arms are observed in spermatid flagella. Sperm flagella assembly does not rely on IFT and is carried out inside the cytoplasm. 
A

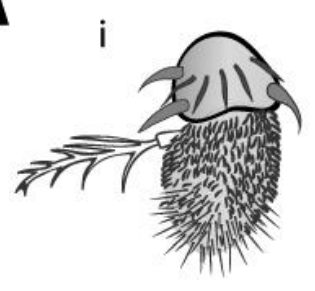

B i
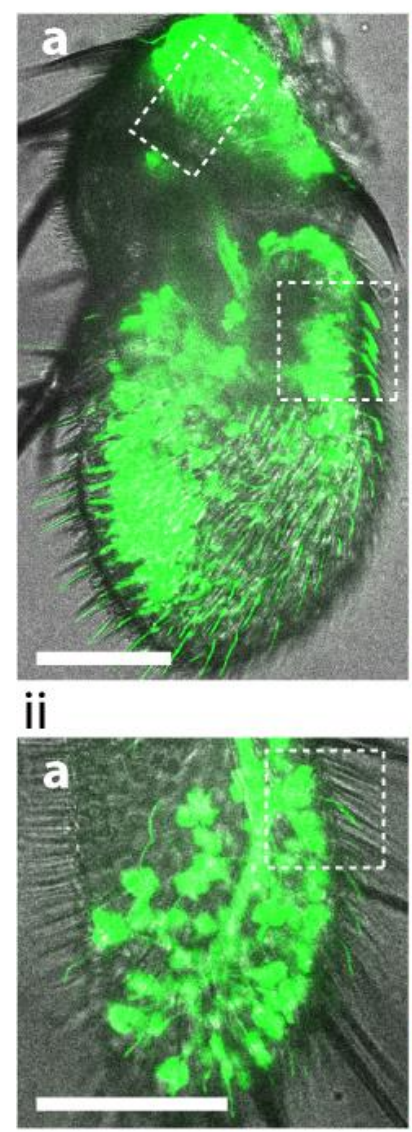

iii

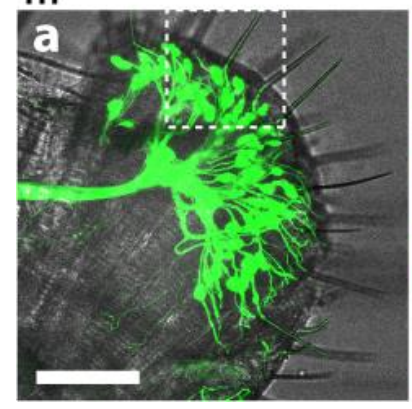

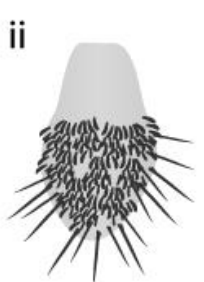
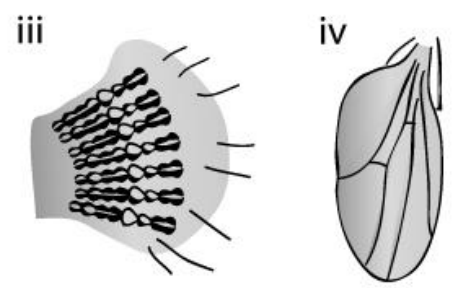

iv
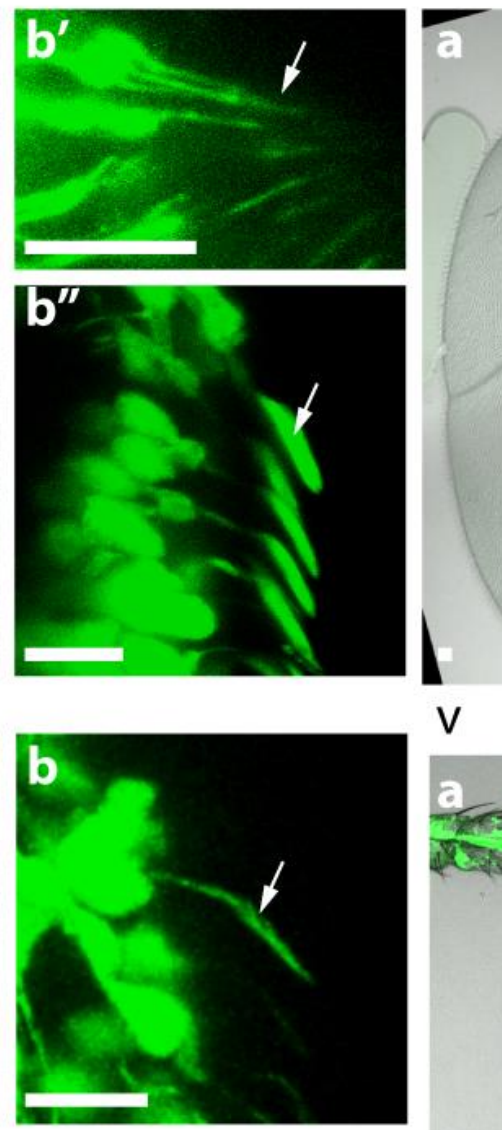

\section{V}

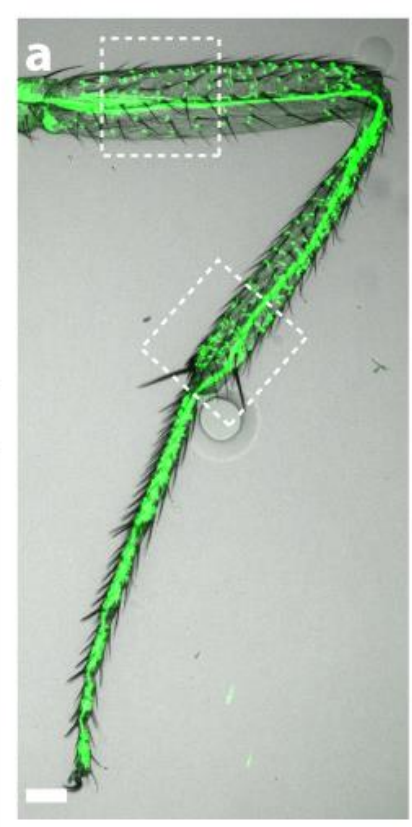

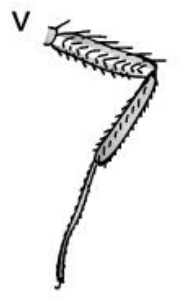
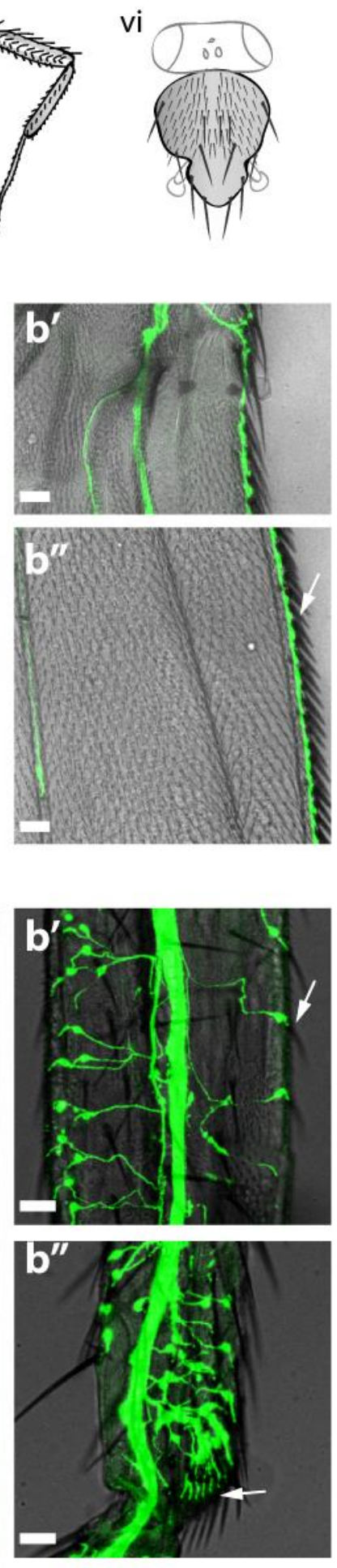

Figure 2: Live imaging of cilia present in many sensory neurons in adult Drosophila. A)

Cartoons of an antenna (i), a maxillary palp (ii), a proboscis (iii), a wing (iv), a leg (v) and a thorax 
(vi) that harbor different types of external sensory neurons. Two antennae, two maxillary palps and one proboscis are present in the fly head. The thorax harbors six legs and two wings. B) Representative images of sensory neurons (marked by UAS-GFP using Ga/4hia) in different external sensory organs, such as second and third antennal segment (i), maxillary palp (ii), proboscis (iii), wing (iv) and leg (v). The bright-field image combined with the fluorescent image of neurons (i-v) helps understanding the organization of the tissue with respect to the cuticle in the given organ. In i-v, a magnified view of the marked region(s) in (a) is presented in (b or b'-b") to highlight the cilia in the sensory neurons. In principle, fluorescently tagged centrosomal components can also be studied. The third antennal segment (i) and maxillary palp (ii) harbor different types of olfactory sensory neurons. Chordotonal neurons (b') are present in the second antennal segment (i). The proboscis (iii) and wing (iv) harbor gustatory, chemo- and mechanosensory neurons, and the leg (v) also harbors mechanosensory neurons. Scale bars represent $50 \mu \mathrm{m}$ in i-a, ii-a, iii-a, iv and v, and $10 \mu \mathrm{m}$ in i-b'-b", ii-b and iii-b. 

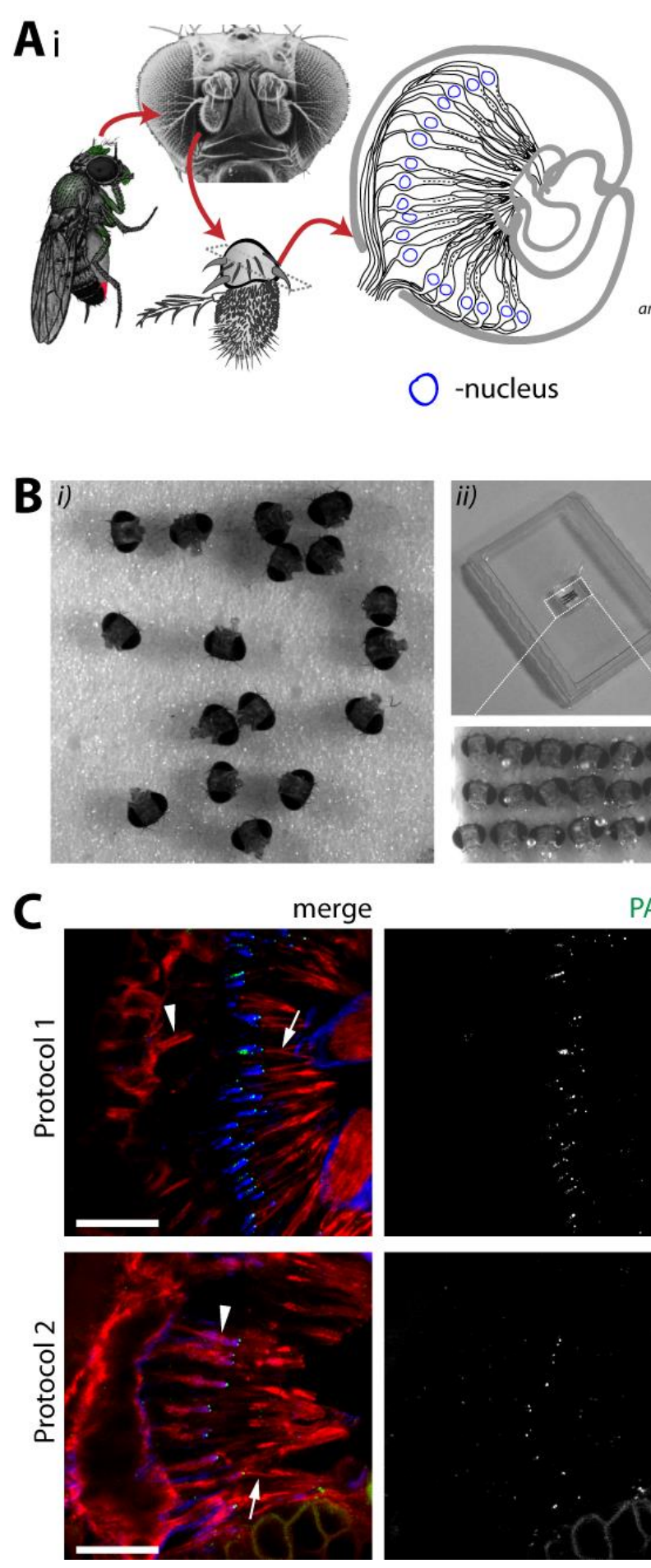
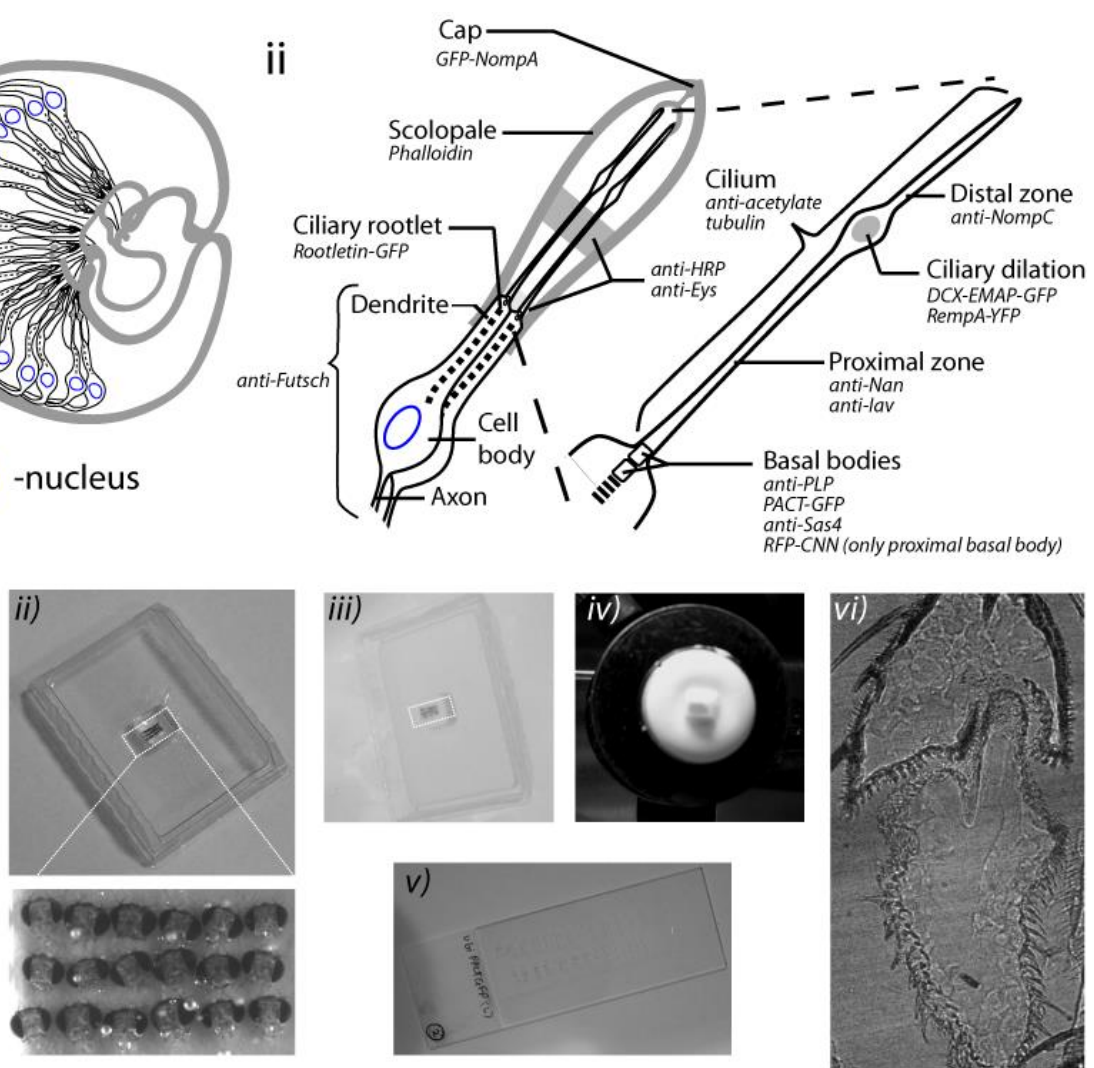

PACT
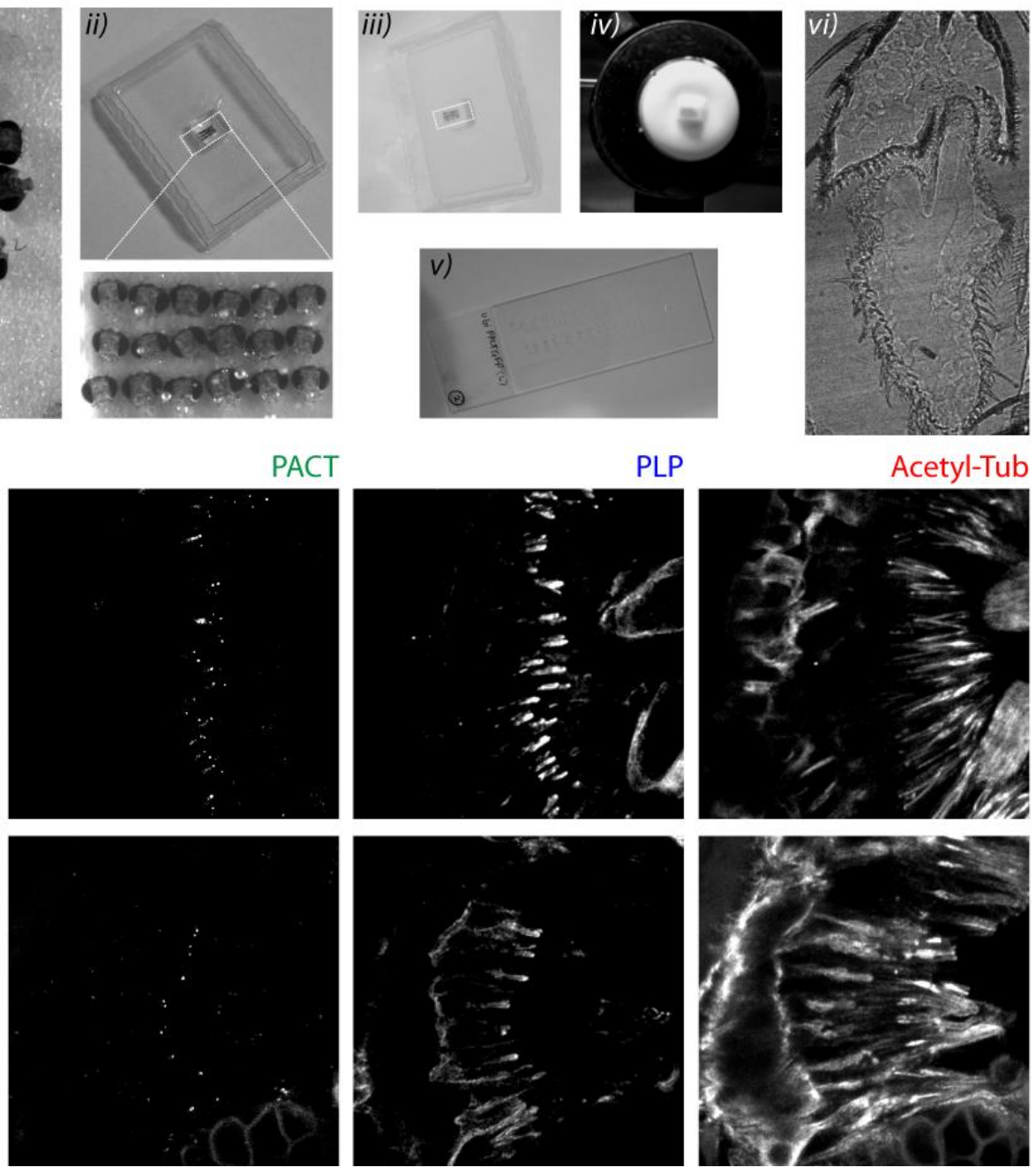

Figure 3: Imaging basal body and cilium in auditory neurons in Drosophila antenna. A) The scheme depicts the organization of (i) the Johnston's organs (JOs) in the second segment of the adult (or pupal) antenna present in the head of the fly, (ii) the chordotonal neurons in the $\mathrm{JO}$ of 
the antenna, and the cilium in those neurons. Some markers for different regions of the centrosomes and cilia are shown (for detailed list of the components of these organelles see Table 1). B) Around 16-20 heads of pupae or 2-4 days old flies are cut (i) followed by aligning heads in a mold filled with OCT. (ii). Then the mold is frozen on dry ice. After the OCT has solidified, the block is mounted upside down (iii) on the cryostat holder (iv). 10-12 $\mu \mathrm{m}$ thin sections are collected on the coated side of the Poly-L-Lysin slide (Sigma, USA). Then a boundary is made using a Dako pen. The boundary acts as a barrier preventing the aqueous solutions from running of the slide during fixing and immunostaining (v). Also see [29].vi) A representative bright-field picture of the second and third antennae in a section. C) Representative pictures of a set of chordotonal neurons in $\mathrm{JO}$ of second antennal segment in which centrioles (PACT in green), pericentriolar material (PLP in red) and cilia (acetylated tubulin in magenta) are marked. Representative images obtained with protocol 1 and 2 are shown (see section 3.1). PACT is the conserved centriole localizing C-terminal domain of pericentrin-like protein (PLP); thus PACT::GFP marks centrioles when expressed in Drosophila. Arrowheads and arrows mark the dendrite and cilia of the auditory neurons, respectively. 


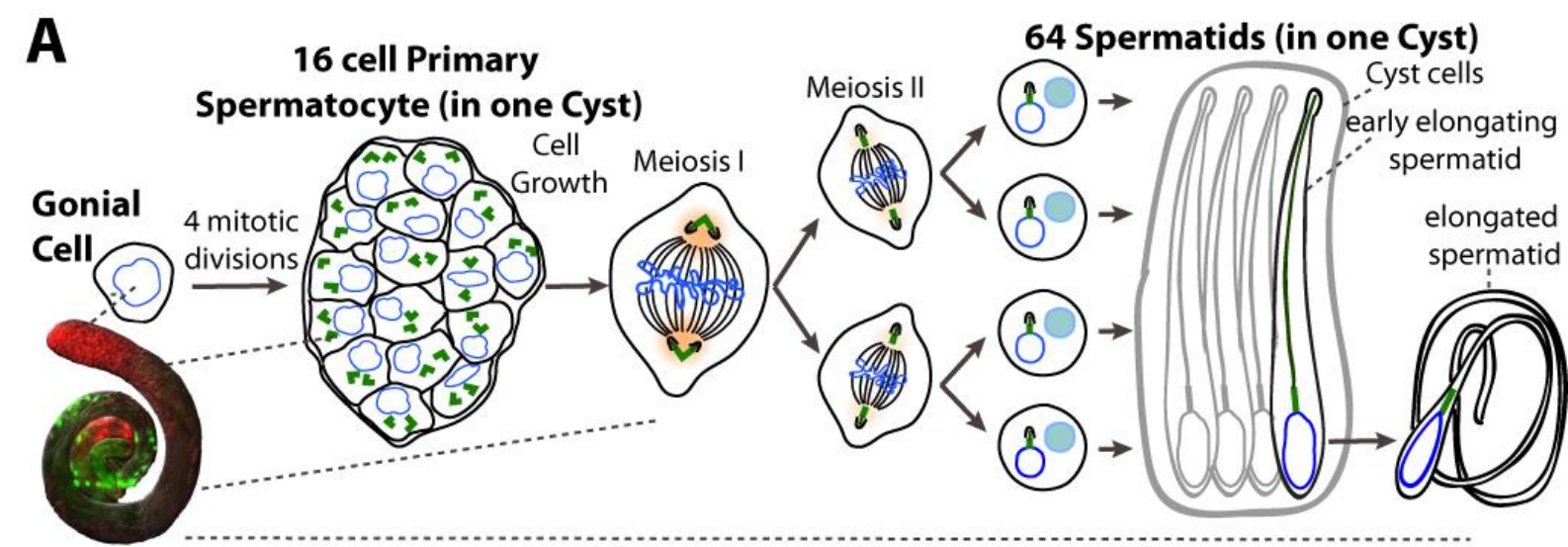

Cell Membrane $\bigcirc$ Nucleus $\mathbb{I}$ Spindle Microtubules $\bigcirc$ Nebenkern

$<$ I Centrioles Pericentriolar Material | Axoneme

B i)

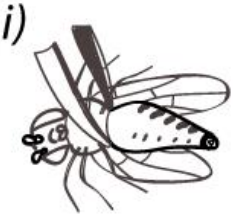

ii)

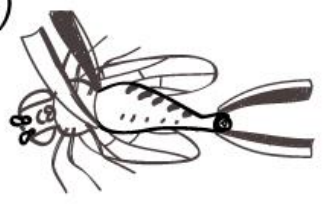

iii)

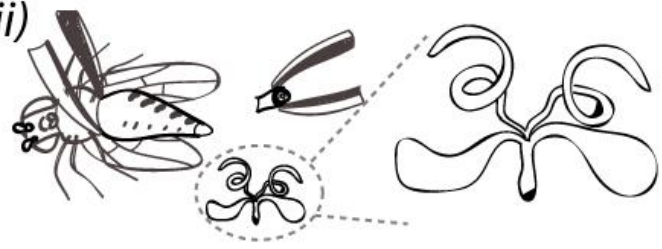

C
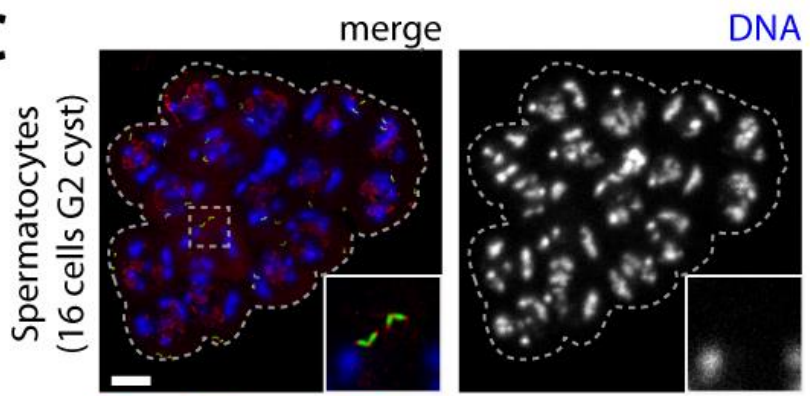

DNA
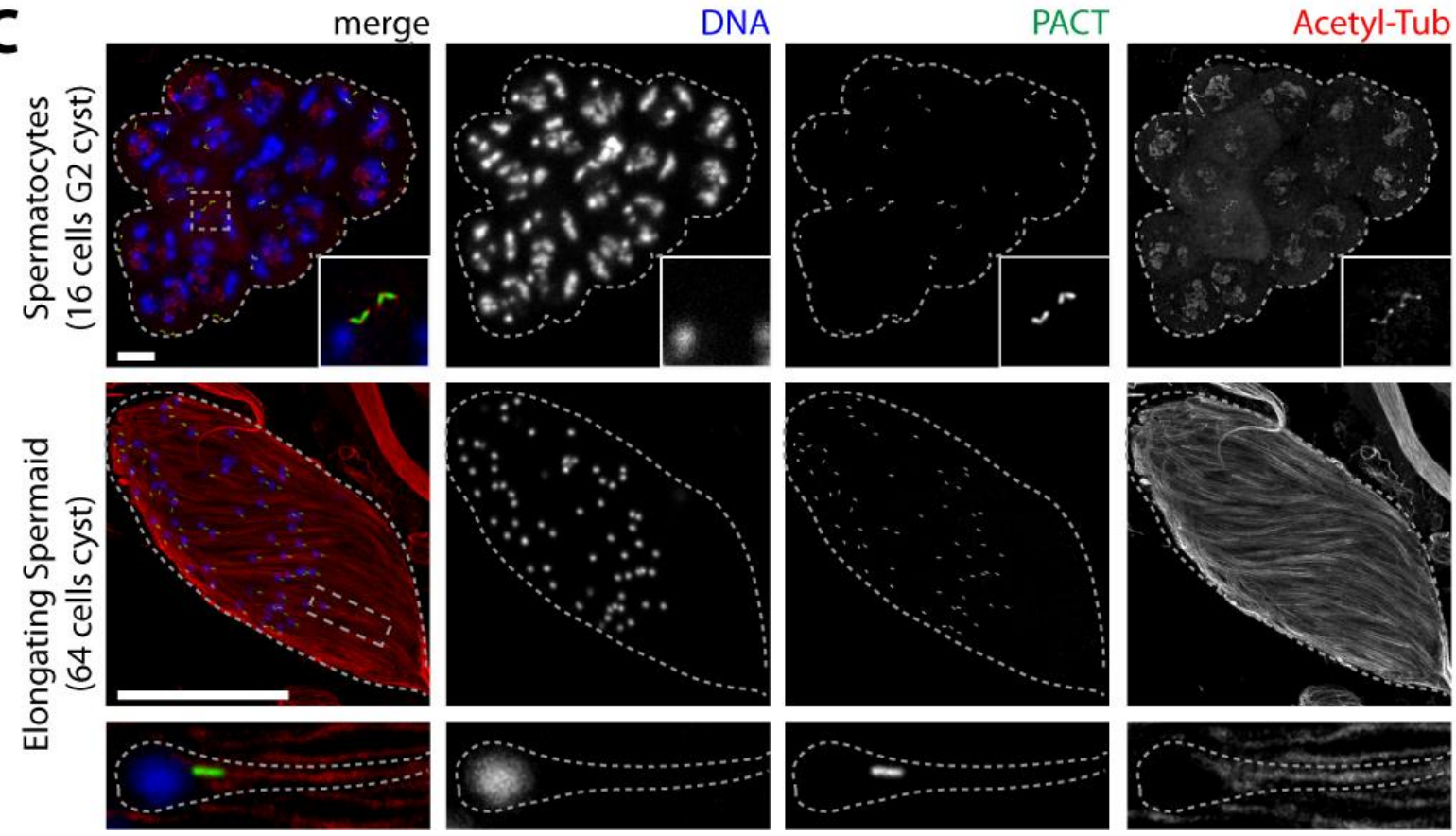

Figure 4: Imaging centriole and cilia in testes in adult (or pupae) Drosophila. A) Schematic representation of Drosophila spermatogenesis. A stem cell after division gives rise to a gonial cell that in turn undergoes four rounds of incomplete mitotic divisions to produce a 16 -cell cyst of 
primary spermatocytes. Primary spermatocytes go through a long G2 phase when centrioles elongate and migrate to the cell membrane where they grow a cilium. Each spermatocyte then undergoes two consecutive meiotic divisions without either DNA replication or centriole duplication. As a result, each early spermatid forms with a single basal body nucleating the sperm flagellum. B) Scheme shows different steps of testes dissection from an adult male fly. Dissection is done in a drop of testis buffer. Hold the fly with legs facing up (i) and pull out the male genital part (ii). Testes will come out with it (iii). If they do not, insert the forceps inside the fly's abdomen and pull them out. Testes can be immunostained either by keeping them intact or squashing them (for details see section 3.2, [30]). C) Representative images of spermatocytes and elongating spermatids from 16 cell G2 and 64 cells cyst, respectively, with markers for DNA (DAPI in blue), centrioles (PACT in green) and cilia (acetylated tubulin in magenta). 
A i)

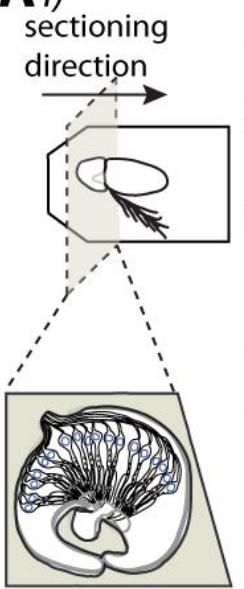

ii)

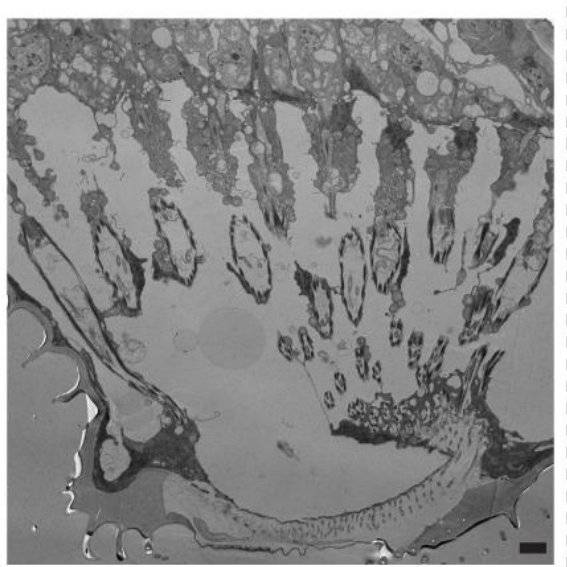

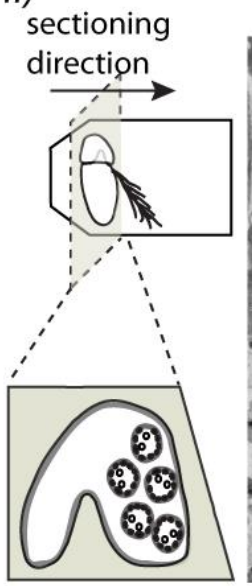

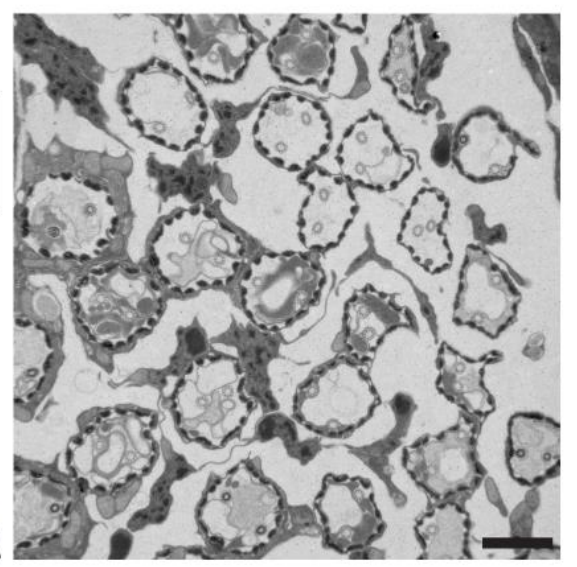

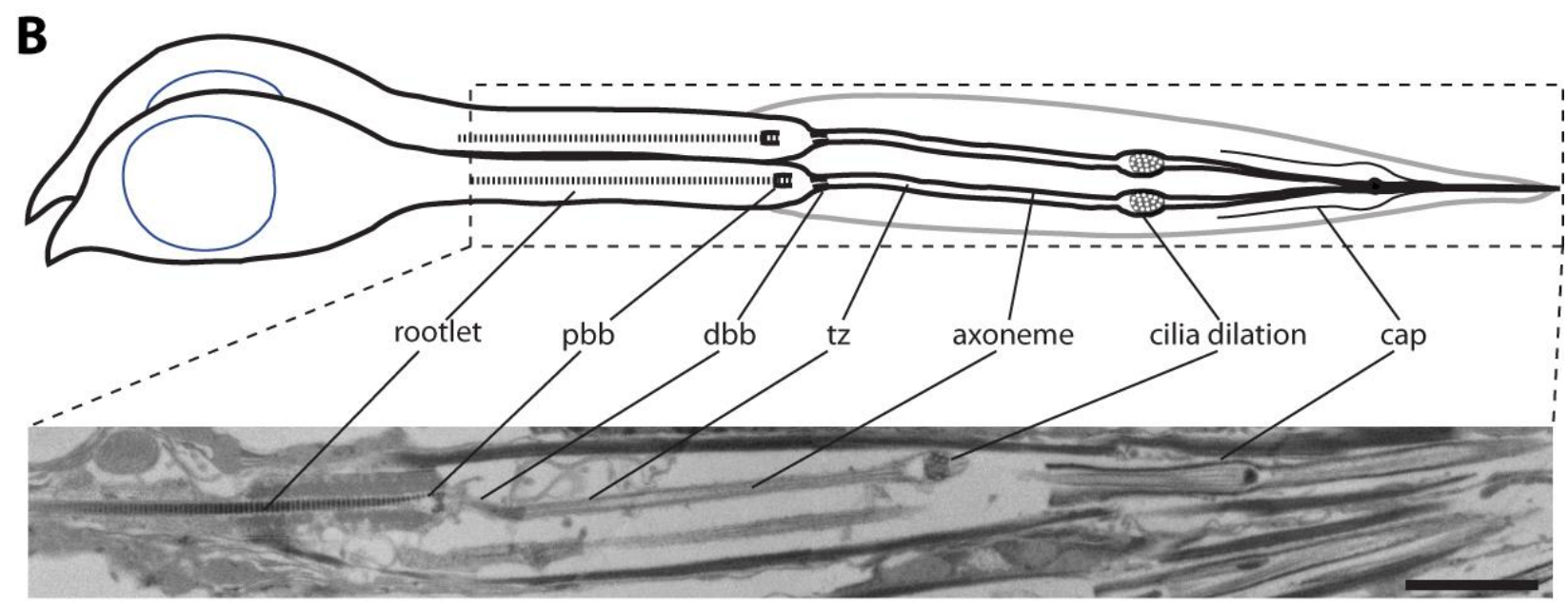

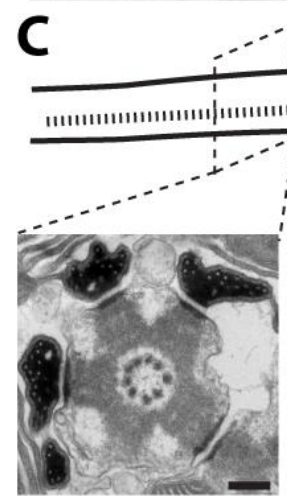

rootlet

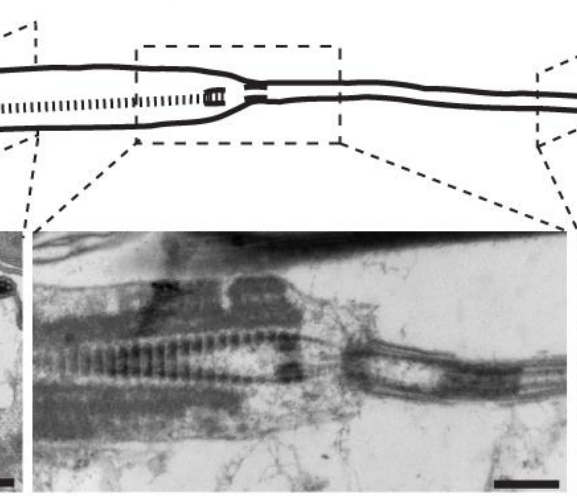

rootlet and transition zone

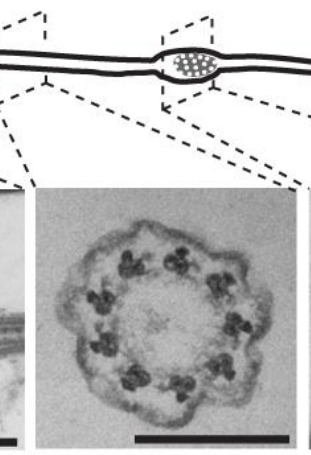

proximal segment

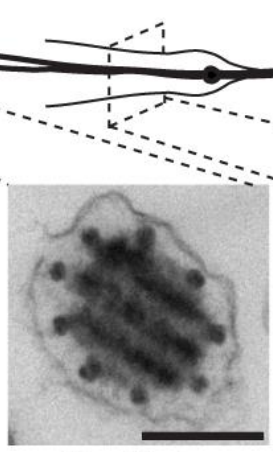

cilia dilation

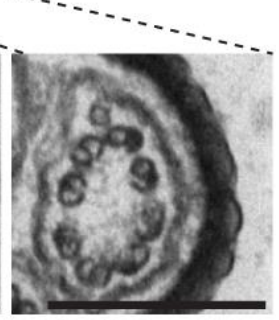

distal segment (in cap)

Figure 5: Electron microscopy analysis of basal body and cilia from auditory neurons. A)

i) Scheme depicts the orientation of the second segment in the antenna in the resin mold and the direction of sectioning to obtain longitudinal sections of chordotonal neurons (or auditory neurons). On the right side of the scheme, a representative low magnification electron micrograph 
displays the longitudinal organization of the Johnston's organs that harbor chordotonal neurons. ii) Scheme represents the orientation of the antennal second segment in the resin mold and the direction of sectioning to achieve cross sections of auditory neurons. On the right side of the scheme, a low magnification electron micrograph representes cross organization of the Johnston's Organ. Scale bars represent $2 \mu \mathrm{m}$ (B) Scheme of the two auditory neurons present in a scolopale and a representative electron micrograph of a longitudinal view of those neurons. Scale bar represents $2 \mu \mathrm{m}$. C) Scheme of the auditory cilium and representative electron micrographs of cross-section and longitudinal view of the different regions of cilia, such as ciliary rootlet, transition zone, the proximal segment of the cilia, ciliary dilation and distal segment of the cilia. Scale bars represent $250 \mathrm{~nm}$. 

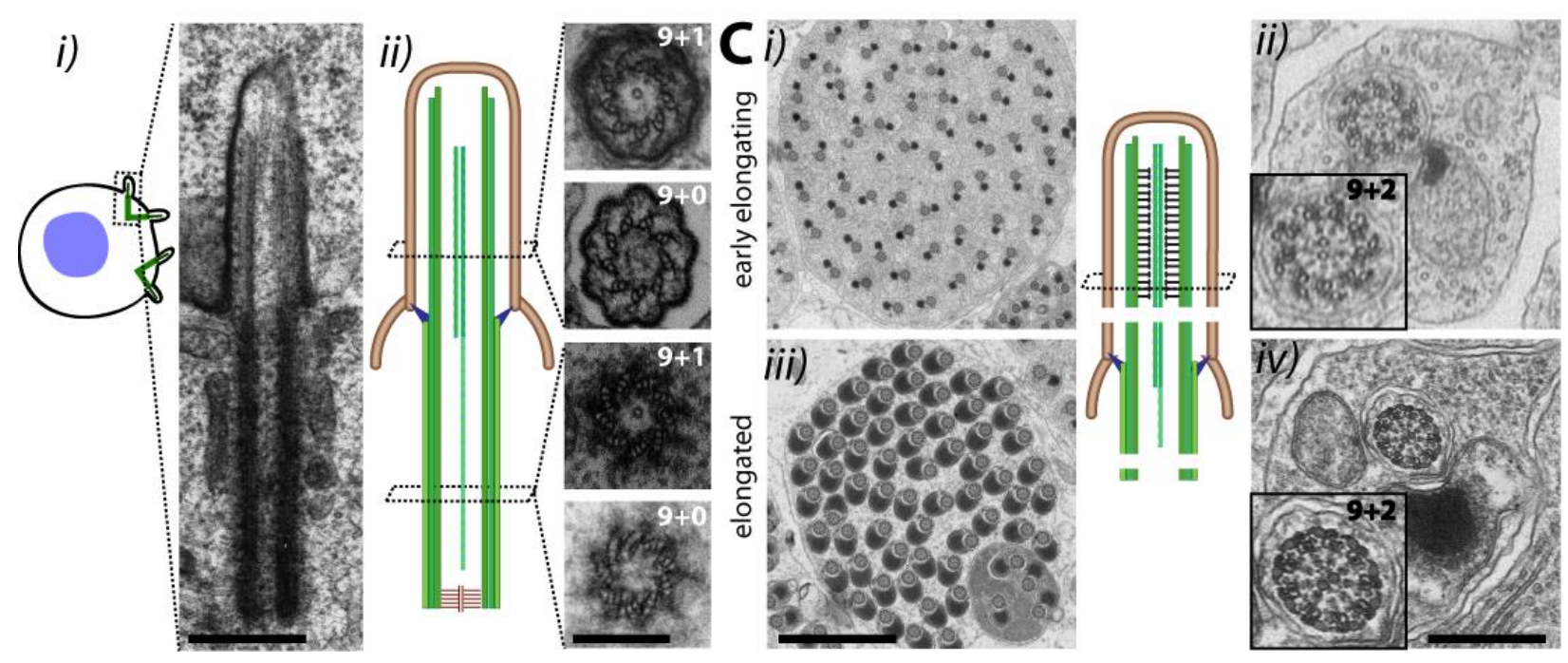

Figure 6: Electron microscopy analysis of centrosomes and cilia from testes. A) Ultrastructural organization of primary cilia-like structure and basal body in G2 stage spermatocyte. i) Longitudinal section of a primary spermatocyte cilium and the basal body. ii) This cilium contains nine radially arranged doublet microtubules either with a central singlet microtubule that extends from basal body to the axoneme $(9+1)$ or without it $(9+0)$. Representative cross section images of axoneme and basal body in both types of cilia are shown. Interestingly, while the basal body is formed with triplet microtubules, the cilium is formed with doublet microtubules. B) Ultrastructural organization of flagella in elongating (i-ii) and elongated (iii-iv) spermatids. Representative images of early elongating (i) and elongated (iii) cysts containing 64 spermatids. Representative cross section images of cilia $(9+2)$ in both types of spermatids are shown. Note changes in axoneme organization during transition from early elongating to elongated spermatid, including the acquisition of dynein arms. 


\section{Tables:}

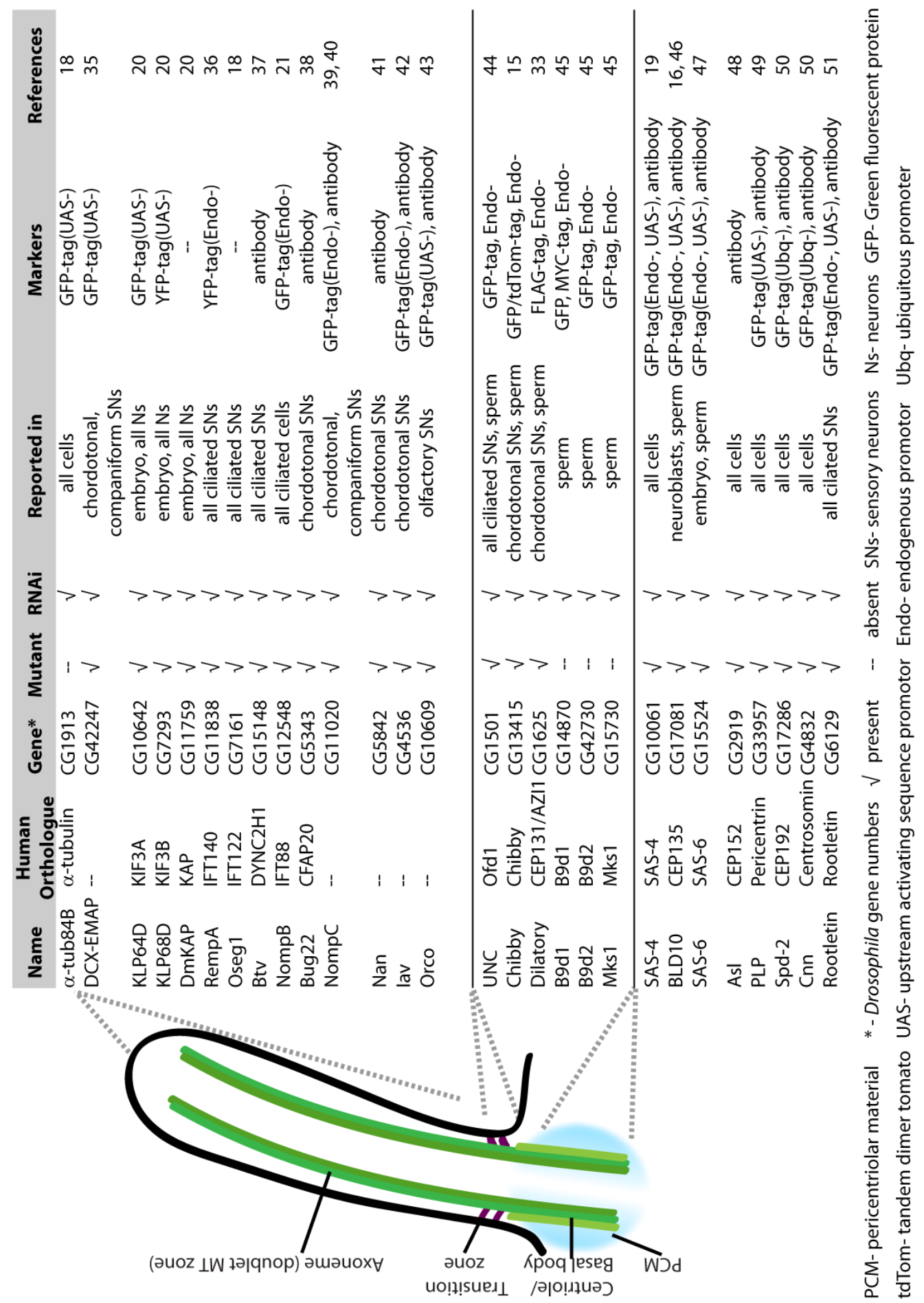

Table 1: Overview of some available tools to study centrosome/ basal body and cilia biology in Drosophila. Note: This table is not an exhaustive list of all centrosome and cilia 
components mapped. Due to space constrain, only references for mutants and/or markers for each gene are mentioned in the list $[15,16,19,21,31,35-51]$. Also, for more information about the genes visit www.flybase.org. 


\section{Acknowledgement:}

We apologize to colleagues whose work was not discussed or cited due to space constraints. We thank the IGC imaging unit (Light microscopy and Electron microscopy) for the help with image acquisition, and the IGC fly facility, and MBD Lab for discussions. SCJ and SW are supported by the FCT (Fundação Portuguesa para a Ciência e Tecnologia) Fellowships SFRH/BPD/87479/2012 and SFRH/BD/52176/2013, respectively. The laboratory and MBD are supported by an EMBO installation grant and an ERC starting grant.

\section{References:}

1. Bettencourt-Dias, M., Hildebrandt, F., Pellman, D., Woods, G., and Godinho, S.A. (2011). Centrosomes and cilia in human disease. Trends Genet 27, 307-315.

2. Jana, S.C., Marteil, G., and Bettencourt-Dias, M. (2014). Mapping molecules to structure: unveiling secrets of centriole and cilia assembly with near-atomic resolution. Curr Opin Cell Biol 26, 96-106.

3. Kozminski, K.G., Beech, P.L., and Rosenbaum, J.L. (1995). The Chlamydomonas kinesin-like protein FLA10 is involved in motility associated with the flagellar membrane. J Cell Biol 131, 1517-1527.

4. Signor, D., Wedaman, K.P., Rose, L.S., and Scholey, J.M. (1999). Two heteromeric kinesin complexes in chemosensory neurons and sensory cilia of Caenorhabditis elegans. Mol Biol Cell 10, 345-360.

5. Dubruille, R., Laurencon, A., Vandaele, C., Shishido, E., Coulon-Bublex, M., Swoboda, P., Couble, P., Kernan, M., and Durand, B. (2002). Drosophila regulatory factor $X$ is necessary for ciliated sensory neuron differentiation. Development 129, 5487-5498.

6. Amack, J.D., and Yost, H.J. (2004). The T box transcription factor no tail in ciliated cells controls zebrafish left-right asymmetry. Curr Biol 14, 685-690.

7. Tonna, E.A., and Lampen, N.M. (1972). Electron microscopy of aging skeletal cells. I. Centrioles and solitary cilia. J Gerontol 27, 316-324.

8. Postlethwait, J.H., and Schneiderman, H.A. (1969). A clonal analysis of determination in Antennapedia a homoeotic mutant of Drosophila melanogaster. Proc Natl Acad Sci U S A 64, 176183.

9. Ursprung, H., Conscience-Egli, M., Fox, D.J., and Wallimann, T. (1972). Origin of leg musculature during Drosophila metamorphosis. Proc Natl Acad Sci U S A 69, 2812-2813.

10. Horikawa, M., and Fox, A.S. (1964). Culture of Embryonic Cells of Drosophila Melanogaster in Vitro. Science 145, 1437-1439.

11. Hotta, Y., and Benzer, S. (1972). Mapping of behaviour in Drosophila mosaics. Nature 240, 527535.

12. Li, K., Xu, E.Y., Cecil, J.K., Turner, F.R., Megraw, T.L., and Kaufman, T.C. (1998). Drosophila centrosomin protein is required for male meiosis and assembly of the flagellar axoneme. J Cell Biol 141, 455-467. 
13. Callaini, G., and Riparbelli, M.G. (1990). Centriole and centrosome cycle in the early Drosophila embryo. J Cell Sci 97 ( Pt 3), 539-543.

14. Mahowald, A.P., and Strassheim, J.M. (1970). Intercellular migration of centrioles in the germarium of Drosophila melanogaster. An electron microscopic study. J Cell Biol 45, 306-320.

15. Enjolras, C., Thomas, J., Chhin, B., Cortier, E., Duteyrat, J.L., Soulavie, F., Kernan, M.J., Laurencon, A., and Durand, B. (2012). Drosophila chibby is required for basal body formation and ciliogenesis but not for Wg signaling. J Cell Biol 197, 313-325.

16. Carvalho-Santos, Z., Machado, P., Branco, P., Tavares-Cadete, F., Rodrigues-Martins, A., PereiraLeal, J.B., and Bettencourt-Dias, M. (2010). Stepwise evolution of the centriole-assembly pathway. J Cell Sci 123, 1414-1426.

17. Carvalho-Santos, Z., Azimzadeh, J., Pereira-Leal, J.B., and Bettencourt-Dias, M. (2011). Evolution: Tracing the origins of centrioles, cilia, and flagella. J Cell Biol 194, 165-175.

18. Avidor-Reiss, T., Maer, A.M., Koundakjian, E., Polyanovsky, A., Keil, T., Subramaniam, S., and Zuker, C.S. (2004). Decoding cilia function: defining specialized genes required for compartmentalized cilia biogenesis. Cell 117, 527-539.

19. Basto, R., Lau, J., Vinogradova, T., Gardiol, A., Woods, C.G., Khodjakov, A., and Raff, J.W. (2006). Flies without centrioles. Cell 125, 1375-1386.

20. Jana, S.C., Girotra, M., and Ray, K. (2011). Heterotrimeric kinesin-II is necessary and sufficient to promote different stepwise assembly of morphologically distinct bipartite cilia in Drosophila antenna. Mol Biol Cell 22, 769-781.

21. Han, Y.G., Kwok, B.H., and Kernan, M.J. (2003). Intraflagellar transport is required in Drosophila to differentiate sensory cilia but not sperm. Curr Biol 13, 1679-1686.

22. Carvalho-Santos, Z., Machado, P., Alvarez-Martins, I., Gouveia, S.M., Jana, S.C., Duarte, P., Amado, T., Branco, P., Freitas, M.C., Silva, S.T., et al. (2012). BLD10/CEP135 is a microtubule-associated protein that controls the formation of the flagellum central microtubule pair. Dev Cell 23, 412424.

23. Riparbelli, M.G., Callaini, G., and Megraw, T.L. (2012). Assembly and persistence of primary cilia in dividing Drosophila spermatocytes. Dev Cell 23, 425-432.

24. Briggs, L.J., Davidge, J.A., Wickstead, B., Ginger, M.L., and Gull, K. (2004). More than one way to build a flagellum: comparative genomics of parasitic protozoa. Curr Biol 14, R611-612.

25. Broekhuis, J.R., Verhey, K.J., and Jansen, G. (2014). Regulation of cilium length and intraflagellar transport by the RCK-kinases ICK and MOK in renal epithelial cells. PLoS One 9, e108470.

26. Blachon, S., Cai, X., Roberts, K.A., Yang, K., Polyanovsky, A., Church, A., and Avidor-Reiss, T. (2009). A proximal centriole-like structure is present in Drosophila spermatids and can serve as a model to study centriole duplication. Genetics 182, 133-144.

27. Gottardo, M., Callaini, G., and Riparbelli, M.G. (2015). The Drosophila centriole - conversion of doublets into triplets within the stem cell niche. J Cell Sci 128, 2437-2442.

28. Martins, A.R., Machado, P., Callaini, G., and Bettencourt-Dias, M. (2010). Microscopy methods for the study of centriole biogenesis and function in Drosophila. Methods Cell Biol 97, 223-242.

29. Vieillard, J., Duteyrat, J.L., Cortier, E., and Durand, B. (2015). Imaging cilia in Drosophila melanogaster. Methods Cell Biol 127, 279-302.

30. Basiri, M.L., Blachon, S., Chim, Y.C., and Avidor-Reiss, T. (2013). Imaging centrosomes in fly testes. J Vis Exp, e50938.

31. Ma, L., and Jarman, A.P. (2011). Dilatory is a Drosophila protein related to AZI1 (CEP131) that is located at the ciliary base and required for cilium formation. J Cell Sci 124, 2622-2630.

32. Noguchi, T., Koizumi, M., and Hayashi, S. (2011). Sustained elongation of sperm tail promoted by local remodeling of giant mitochondria in Drosophila. Curr Biol 21, 805-814.

33. Kavlie, R.G., and Albert, J.T. (2013). Chordotonal organs. Curr Biol 23, R334-335. 
34. Mishra, M. (2015). A quick method to investigate the Drosophila Johnston's organ by confocal microscopy. Journal of Microscopy and Ultrastructure 3, 1-7.

35. Bechstedt, S., Albert, J.T., Kreil, D.P., Muller-Reichert, T., Gopfert, M.C., and Howard, J. (2010). A doublecortin containing microtubule-associated protein is implicated in mechanotransduction in Drosophila sensory cilia. Nat Commun 1, 11.

36. Lee, E., Sivan-Loukianova, E., Eberl, D.F., and Kernan, M.J. (2008). An IFT-A protein is required to delimit functionally distinct zones in mechanosensory cilia. Curr Biol 18, 1899-1906.

37. Eberl, D.F., Hardy, R.W., and Kernan, M.J. (2000). Genetically similar transduction mechanisms for touch and hearing in Drosophila. J Neurosci 20, 5981-5988.

38. Mendes Maia, T., Gogendeau, D., Pennetier, C., Janke, C., and Basto, R. (2014). Bug22 influences cilium morphology and the post-translational modification of ciliary microtubules. Biol Open 3, 138-151.

39. Walker, R.G., Willingham, A.T., and Zuker, C.S. (2000). A Drosophila mechanosensory transduction channel. Science 287, 2229-2234.

40. Liang, X., Madrid, J., Saleh, H.S., and Howard, J. (2011). NOMPC, a member of the TRP channel family, localizes to the tubular body and distal cilium of Drosophila campaniform and chordotonal receptor cells. Cytoskeleton (Hoboken) 68, 1-7.

41. Newton, F.G., zur Lage, P.I., Karak, S., Moore, D.J., Gopfert, M.C., and Jarman, A.P. (2012). Forkhead transcription factor Fd3F cooperates with $\mathrm{Rfx}$ to regulate a gene expression program for mechanosensory cilia specialization. Dev Cell 22, 1221-1233.

42. Gong, Z., Son, W., Chung, Y.D., Kim, J., Shin, D.W., McClung, C.A., Lee, Y., Lee, H.W., Chang, D.J., Kaang, B.K., et al. (2004). Two interdependent TRPV channel subunits, inactive and Nanchung, mediate hearing in Drosophila. J Neurosci 24, 9059-9066.

43. Benton, R., Sachse, S., Michnick, S.W., and Vosshall, L.B. (2006). Atypical membrane topology and heteromeric function of Drosophila odorant receptors in vivo. PLoS Biol 4, e20.

44. Baker, J.D., Adhikarakunnathu, S., and Kernan, M.J. (2004). Mechanosensory-defective, malesterile unc mutants identify a novel basal body protein required for ciliogenesis in Drosophila. Development 131, 3411-3422.

45. Basiri, M.L., Ha, A., Chadha, A., Clark, N.M., Polyanovsky, A., Cook, B., and Avidor-Reiss, T. (2014). A migrating ciliary gate compartmentalizes the site of axoneme assembly in Drosophila spermatids. Curr Biol 24, 2622-2631.

46. Mottier-Pavie, V., and Megraw, T.L. (2009). Drosophila bld10 is a centriolar protein that regulates centriole, basal body, and motile cilium assembly. Mol Biol Cell 20, 2605-2614.

47. Rodrigues-Martins, A., Bettencourt-Dias, M., Riparbelli, M., Ferreira, C., Ferreira, I., Callaini, G., and Glover, D.M. (2007). DSAS-6 organizes a tube-like centriole precursor, and its absence suggests modularity in centriole assembly. Curr Biol 17, 1465-1472.

48. Dzhindzhev, N.S., Yu, Q.D., Weiskopf, K., Tzolovsky, G., Cunha-Ferreira, I., Riparbelli, M., Rodrigues-Martins, A., Bettencourt-Dias, M., Callaini, G., and Glover, D.M. (2010). Asterless is a scaffold for the onset of centriole assembly. Nature 467, 714-718.

49. Galletta, B.J., Guillen, R.X., Fagerstrom, C.J., Brownlee, C.W., Lerit, D.A., Megraw, T.L., Rogers, G.C., and Rusan, N.M. (2014). Drosophila pericentrin requires interaction with calmodulin for its function at centrosomes and neuronal basal bodies but not at sperm basal bodies. Mol Biol Cell 25, 2682-2694.

50. Conduit, P.T., and Raff, J.W. (2015). Different Drosophila cell types exhibit differences in mitotic centrosome assembly dynamics. Curr Biol 25, R650-651.

51. Laurencon, A., Dubruille, R., Efimenko, E., Grenier, G., Bissett, R., Cortier, E., Rolland, V., Swoboda, P., and Durand, B. (2007). Identification of novel regulatory factor X (RFX) target genes by comparative genomics in Drosophila species. Genome Biol 8, R195. 